\title{
Extinction under intracranial and conventional reinforcement: Effect of a correlated counter
}

\section{JOSEPH P. HUSTON, DEPARTMENT OF PSYCHOLOGY, TUFTS UNIVERSITY, Medford, Mass. 02155}

Rats underwent fixed-ratio training and extinction with and without a correlated counter under either intracranial or liquid reinforcement. The added counter increased resistance to extinction. This effect was at tributed to secondary reinforcing properties of the counter, and taken as evidence for an interpretation of rapid extinction under ICR as being due to lack of conditioned reinforcement.

Comparisons between ICR (intracranial reinforcement) and conventional reinforcers have led to the generalization that intracranial self-stimulation undergoes extinction more rapidly than conventionally reinforced behavior.

Deutsch has accounted for the quick extinction with his drive-decay theory which explains the rapid cessation of responding as resulting from the rapid decay of an electrically induced drive (Deutsch \& Howarth, 1963). Several studies have shown, however, that the rapid extinction may in part be attributable to situational factors, and that when the contingencies of reinforcement are made more similar, differences in resistance to extinction decrease. For instance, McIntire \& Wright (1965) increased resistance to extinction when ICR was made contingent on the rat's pressing a bar plus subsequently touching a water cup. In a similar experiment, Gibson, et al (1965) found no differences in extinction between groups which were reinforced with water or ICS immediately upon licking a dipper. These studies support an interpretation of rapid extinction as resulting from lack of conditioned reinforcement in the usual Skinner-box situation. Whereas conventional reinforcers presented in the Skinner-box allow for conditioned reinforcement to occur by providing external conditioned goal-stimuli, such as the food pellet, dipper, etc., and requiring consummatory response sequences, intracranial reinforcement has no such external referent, and involves no consummatory activity. Furthermore, the temporal contingency between bar press and ICR provides minimal opportunity for conditioned reinforcement.

This hypothesis was tested by adding a correlated counter to the Skinner-box (Ferster \& Skinner, 1957). It was predicted that the correlated counter situation would increase resistance to extinction under ICR since it provides the delay of reinforcement, the external referent stimuli, and the instrumental lever responses, all of which are presumably necessary for the establishment of conditioned reinforcement.

Method. The apparatus was a Skinner-box with an added counter. The counter consisted of a motor-driven white belt the edge of which faced a window which was cut into the Skinner-box. Onto the belt were attached two upright black $2 \times 3$ in. squares which were so spaced that only one was in view from the Skinner-box at any time. At the beginning of a fixed-ratio run one of the squares was 25 in. distant from the window. Under a FR schedule of 15:1, each lever press advanced the square $1 / 15$ of its distance toward the window; upon the fifteenth response reinforcement followed, the square disappeared under the roller of the belt, and the next stimulus was brought into view. The situation is likened to a runway except that the goal approaches the $\mathbf{S}$ whose behavior is monitored with a cumulative recorder. For a diagram and more detailed description of the apparatus see Huston (1967).

Each lever press required $20 \mathrm{~g}$ pressure and delivered either a 15 weight per cent sucrose solution or intracranial stimulation consisting of sinusoidal 60 $\mathrm{Hz}$ pulses with pulse-train durations set at $0.5 \mathrm{sec}$ for all Ss. Stimulus parameters were monitored with an oscilloscope.

Three-month-old male rats of the Long Evans strain were implanted with bipolar electrodes aimed at regions along the medial forebrain bundle. Subsequent histology showed the placements of the positive $S$ s to lie in the anterior lateral hypothalamic region. Eight positive Ss were used for the ICR groups, and eight other implanted rats were used for the comparison groups under SWR (sugar water reinforcement).
Training and Extinction. The ICR and SWR Ss received 2500 reinforcements over 10 days of training and were extinguished. They were then divided into two subgroups; four $S s$ in each group were chosen for training under a FR 15:1 schedule with conditioned reinforcement by means of the correlated counter; these groups are designated FR 15-ICR-Belt and FR 15-SWR-Belt, respectively. The other four $S s$ in each group were trained under a FR 15:1 schedule in the Skinner-box without the correlated counter, and are designated FR I5-ICR-No Belt and FR 15-SWR-No Belt. Animals were run in random order each day. Most Ss could be shaped to FR 15:1 during the first day of training. Stimulus amplitude was adjusted individually and ranged from 200 to $400 \mu \mathrm{A}$, base to peak. Each group received seven days of training, which amounted to a total of 700 reinforcements for each S. Each session was terminated after 100 reinforcements. After each session the SWR rats were allowed access to a water-bottle for about $1 \mathrm{~min}$. All rats were fed after each session, enough to maintain their weight at about $85 \%$. On the eighth day each $S$ underwent extinction training after 40 reinforcements. Extinction was recorded for $1 \mathrm{~h}$. It was necessary to gather all the data for the ICR groups prior to running the SWR comparisons three weeks later; this must be considered when interpreting the data.

Results and Discussion. For purposes of analysis the total number of responses emitted during consecutive 2 -min periods in extinction was used as raw data. Figure 1 summarizes the extinction data for the four groups in blocks of five 2-min periods. It also indicates the mean pre-extinction response rates of each group. A Lindquist Type III analysis of variance revealed that the FR 15 SWR groups were more resistant to extinction than the FR 15 ICR groups, and the two Belt (conditioned reinforcement) groups were more resistant to extinction than the No Belt groups ( $p<.025$ for both). As predicted the FR 15-ICR-Belt rats emitted more responses to extinction than the FR 15-ICR-No Belt group (1747 vs 991); furthermore, the difference between the number of responses emitted by the FR 15-ICR-Belt and FR 15-SWR-No Belt groups is minimal (1747 vs 1874). An unexpected result was that the FR 15-SWR-Belt group gave more responses than the FR 15-SWR-No Belt group. Table 1 Column A lists the total number of responses emitted during extinction by each group. A Newman Keuls test for differences between individual group totals confirmed these differences; all groups differed from each other $(p<.01)$ except groups FR 15-ICR-Belt and FR 15-SWR-No Belt. To summarize, for both water and intracranial reinforcement the correlated counter increased resistance to extinction; also there was no difference between the ICR group with the counter and the SWR group without the counter.

In order to control for effects of differences in acquisition performance on rate of responding during extinction, the data from each rat were transformed into percentages of response rate emitted during acquisition; i.e., each animal's raw extinction

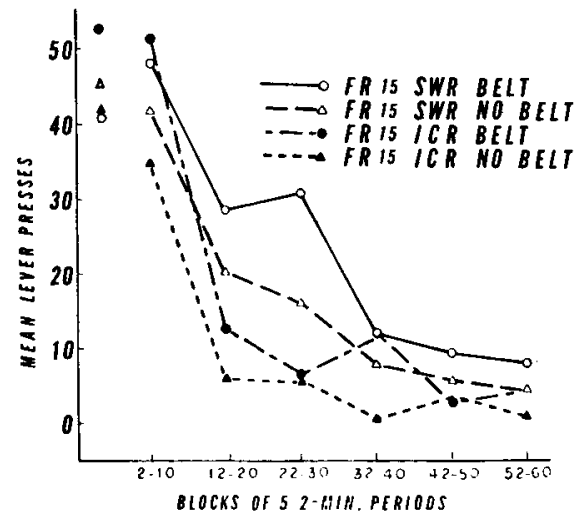

Fig. 1. Mean number of responses per 2 min of extinction over blocks of five 2 -min periods with $N=4$. 
Table 1

Performance During Extinction Expressed as Total Number of Responses (Column A), and as Totals of Percentage Scores (Column B).

\begin{tabular}{cc} 
A & B \\
Total No. & Total of \\
Responses & \% Scores \\
\hline
\end{tabular}

\begin{tabular}{lrr}
\hline h FR $15(\mathrm{~N}=4)$ & & \\
SWR - Belt & 2717 & 3251 \\
SWR - No Belt & 1874 & 2108 \\
ICR - Belt & 1747 & 1794 \\
ICR - No Belt & 991 & 1260 \\
$1 / 2$ h CRF $(\mathrm{N}=8)$ & & \\
CRF - SWR & 1507 & \\
CRF - ICR & 917 & \\
\hline
\end{tabular}

scores (number of responses during successive 2 -min periods) were divided by its mean terminal response rate (mean number of responses per 2 min emitted prior to the extinction session). The totals of the transformed scores are summarized in Table 1 Column B. An analysis of variance performed on the percentage scores showed that the same main effects were significant as in the above analysis ( $p<.01$ for both); the Newman Keuls test gave the same results as above, except that this time the total of the FR 15-ICR-Belt group was significantly less than that of the FR 15-SWR-No Belt group. Since this difference was not significant in the raw score analysis it indicates that pre-extinction rate influences responses emitted during extinction, and that the ICR-Belt rats had higher terminal response rates than the SWR-No Belt rats. Hence, when pre-extinction performance is controlled for, i.e., when comparative rates of extinction instead of absolute number of responses during extinction are taken as the appropriate measure, each water reinforced group is more resistant to extinction than each ICR group. However, the correlated counter still increased resistance to extinction.

While not focal to the problem, some cumulative records were obtained under ICR with the added counter. Ratios up to FR 60 were readily established. It is of interest that the ratio runs were generally positively accelerated curves resembling the curves characteristic of conventionally reinforced ratio behavior; also, they tended to exhibit the postreinforcement pause. This has some bearing on Deutsch's drive decay theory from which one might predict an increase in response rate following each reinforcement rather than a pause and casual onset, if the stimulation induces a drive which attenuates over time.

The experiment has shown that ICR rats, when trained and extinguished with an added counter or goal stimulus, are more resistant to extinction than when trained without the counter. In so far as this effect can be attributed to the secondary reinforcing properties of the goal stimulus, an interpretation of the rapid extinction under ICR in terms of lack of conditioned reinforcement was supported. The lack of conditioned reinforcement hypothesis can also account for difficulties that have been reported in establishing self-stimulation under intermittent reinforcement schedules, and is in accord with the data of Gibson, et al (1965), McIntire \& Wright (1965), and the study by Olds (1956) which found no differences in extinction between food and ICR using a maze.

\section{REFERENCES}

DEUTSCH, J. A., \& HOWARTH, C. I. Some tests of a theory of intracranial self-stimulation. Aychol Rev., 1963, 70, 444-460.

FERSTER, C. B., \& SKINNER, B. F. Schedules of reinforcement. New York: Appleton-Century-Crofts, 1957.

GIBSON, W. E., REID, L. D., SAKAI, M., \& PORTER, D. G. Intracranial reinforcement compared with sugar-water reinforcement. Science, 1965 , $148,1357-1358$.

HUSTON, J. P. A simulated runway with an approaching goal and stationary subject. Psychol. Rep., 1967, 21, 871-872.

LINDQUIST, E. F. Design and analysis of experiments in psychology and education. Boston: Houghton Mifflin Co., 1953.

McINTIRE, R. W., \& WRIGHT, J. E. Differences in extinction in electrical brain-stimulation under traditional procedures of reward presentation. Psychol. Rep., 1965, 16, 909-913.

OLDS, J. Runway and maze behavior controlled by baso-medial forebrain stimulation in the rat. J. comp. physiol. Psychol, 1956, 49, 507-512.

SIDMAN, M., BRADY, J. V., BOREN, J. J., \& CONRAD, D. G. Reward schedules and behavior maintained by intracranial self-stimulation. Science, $1955,122,830-832$.

\section{NOTE}

1. Based on part of a Masters Thesis, Tufts University, June, 1967. Thanks is due to committee chairman A. William Mills and members Bernard W. Harleston, Ina Samuels, and Ezra V. Saul. 